\title{
An analysis of the barbell motion depending on its weight in disabled powerlifting
}

Authors' Contribution:

A - Study Design

B - Data Collection

C - Statistical Analysis

D - Data Interpretation

E - Manuscript Preparation

$F$ - Literature Search

$\mathrm{G}$ - Funds Collection
Wojciech Seidel ${ }^{1 \text { ABCDEF }}$, Anna Zurowska ${ }^{2 \text { DEF }}$

${ }^{1}$ University School of Physical Education in Wroclaw, Poland

${ }^{2}$ Angulus Silesius State School of Higher Vocational Education in Walbrzych, Poland

Key words: disabled powerlifting, powerlifting biomechanics, motion analysis.

Background:

Material/Methods:

Results:

Conclusions:

\section{Abstract}

The aim of the study was to assess the symmetry of flexion and extension movements in upper limbs in disabled powerlifters during bench press with different barbell loads.

29 disabled athletes from the National Powerlifting Team were examined. Mean age was between $23.9 \pm 6.1$. Mean sport experience was $5.4 \pm 3.6$ years.

Both flexion and extension movement were performed symmetrically, which was indicated by an insignificant $(p<0.05)$ difference in the movement onset in the left and the right upper limb.

Time differences in the movement onset and the achievement of maximal angular velocity were statistically insignificant in all trials (loads from $40 \%$ to $95 \% 1 R M$ ). During extension with loads over $60 \% 1 R M$ differences in time of achieving maximal angular velocity were statistically significant or close.

Word count: 1,621

Tables: 0

Received: April 2013

Figures: 4

Accepted: July 2014

References: 15

Published: October 2014

Corresponding author:

Wojciech Seidel Ph.D.

University School of Physical Education

Dep. of Disability Sport

al. I.J. Paderewskiego 35, bud. P4 pok. 2/47, 51-612 Wrockaw

E-mail: wojciech.seidel@awf.wroc.pl 


\section{Introduction}

Disabled powerlifting is a Paralympic discipline mainly based on strength training which is commonly practiced by disabled athletes, not only powerlifters. In powerlifting an athlete performs a bench press (lowering the barbell towards the chest and pressing it upwards until full elbow extension) in a supine position. The movement can be described as flexion and extension of the upper limbs in shoulder and elbow joints.

During each bench press the athlete must generate strength both during flexion (downward barbell motion) and extension (upward barbell motion) in order to overcome the barbell load. Generating strength in different time periods can be seen as an important factor influencing untimely fatigue, injuries resulting from overload of one upper limb and incorrect technique [2, 9]. The athlete should perform the movement controlling both the velocity and therange of motion in shoulder and elbow joints. During flexion muscles work eccentrically, while during extension concentrically. A change in the direction of movement does not allow the utilization of additional energy coming from the stretch reflex. This situation results from the rules which state that the barbell must stop on the chest. Therefore, the complete barbell motion must be considered as two independent biomechanical movements.

It can be suspected that the movement asymmetry will increase with the barbell load [9]. A reduced ability to generate power in both upper limbs in comparison to one limb was extensively studied in different tasks such as: lower limbs extension [5, 6, 8, 14, 15], foot dorsiflexion [12], elbow flexion and extension [7, 11, 13], wrist extension and handgrip [11, 13]. However, in some of those the asymmetry was insignificant $[11,14]$ or negative. It was observed that power achieved by both upper limbs working simultaneously was greater than the sum of two upper limbs working separately [8]. It was also dependent on the examined population [14], task [6] and body parts [11].

Although asymmetry in different movements was extensively studied, there are few studies regarding functional and dynamic asymmetry considered as a factor determining the training process progress of disabled athletes. It seems that this knowledge regarding asymmetry can be crucial for coaching but also useful in teaching symmetrical physical activities.

Assuming that one criterion of a correct bench press is the symmetry of movement, it can be supposed that some stages of the bench press should be performed simultaneously. If this does not happen, it will be possible to determine the load that caused the least asymmetry.

The aim of the study was to assess the symmetry of flexion and extension movements in upper limbs in disabled powerlifters during bench press with different barbell loads.

\section{Material and methods}

Material. 29 disabled athletes from the National Powerlifting Team were examined. The mean age was between $23.9 \pm 6.1$, which the mean sport experience was $5.4 \pm 3.6$ years.

All subjects had normal upper limbs function. Powerlifting as a Paralympic sport is open to athletes with the following eight eligible physical impairments (impaired muscle power, impaired passive range of movement, limb deficiency, leg length difference, short stature, hypertonia, ataxia, and athetosis) with a range of physical disabilities, including (cerebral palsy, spinal cord injuries, lower limb amputation, poliomyelitis), who meet the current minimal eligibility criteria and can perform, safely and appropriately, according to the IPC Powerlifting rules. All eligible athletes compete in one sport class, but in different weight categories.

Method. The study was conducted in January 2010 at the gym of the University School of Physical Education in Wroclaw, Education Center for the Disabled in Wroclaw and Sport Center "Zawisza" in Bydgoszcz.

The athletes performed a bench press with 40\%1RM, 60\%1RM, 80\%1RM and 95\%1RM (1RM - one repetition maximum) in a supine position. There was a 3-minute rest between successive attempts. 1RM for each athlete was established the day before according to Dahoney and Dias procedure [3, 4]. The maximal grip width was determined by arm adduction of $90^{\circ}$ in the frontal plane and elbows bent at $90^{\circ}$.

Equipment. In order to evaluate the movement symmetry two uniaxial potentiometric goniometers, placed on both elbow joints, were used. The goniometer arm coincided with the 
upper arm and the forearm long axis. The axis of rotation of the goniometer coincided with the elbow joint axis. The onset of flexion and extension movement was determined based on recording of angle changes in the elbow joint as a function of time. It also allowed determining the time point of maximal angular velocity during flexion and extension.

Data processing. Based on the data obtained from goniometers, angular velocities in the right and left elbow joint were calculated. The onset of movement and time point of maximum angular velocity in the right and left elbow joint during flexion and extension were determined. Based on that the value difference of onset time and maximal angular velocities were calculated (Fig. 1). A greater value of the difference indicated asymmetry, and a lower value indicated that the movement was performed more symmetrically. A positive value of the time difference meant that the left upper limb movement began earlier than the right one, and the negative difference value that the left upper limb movement began later than the right one.

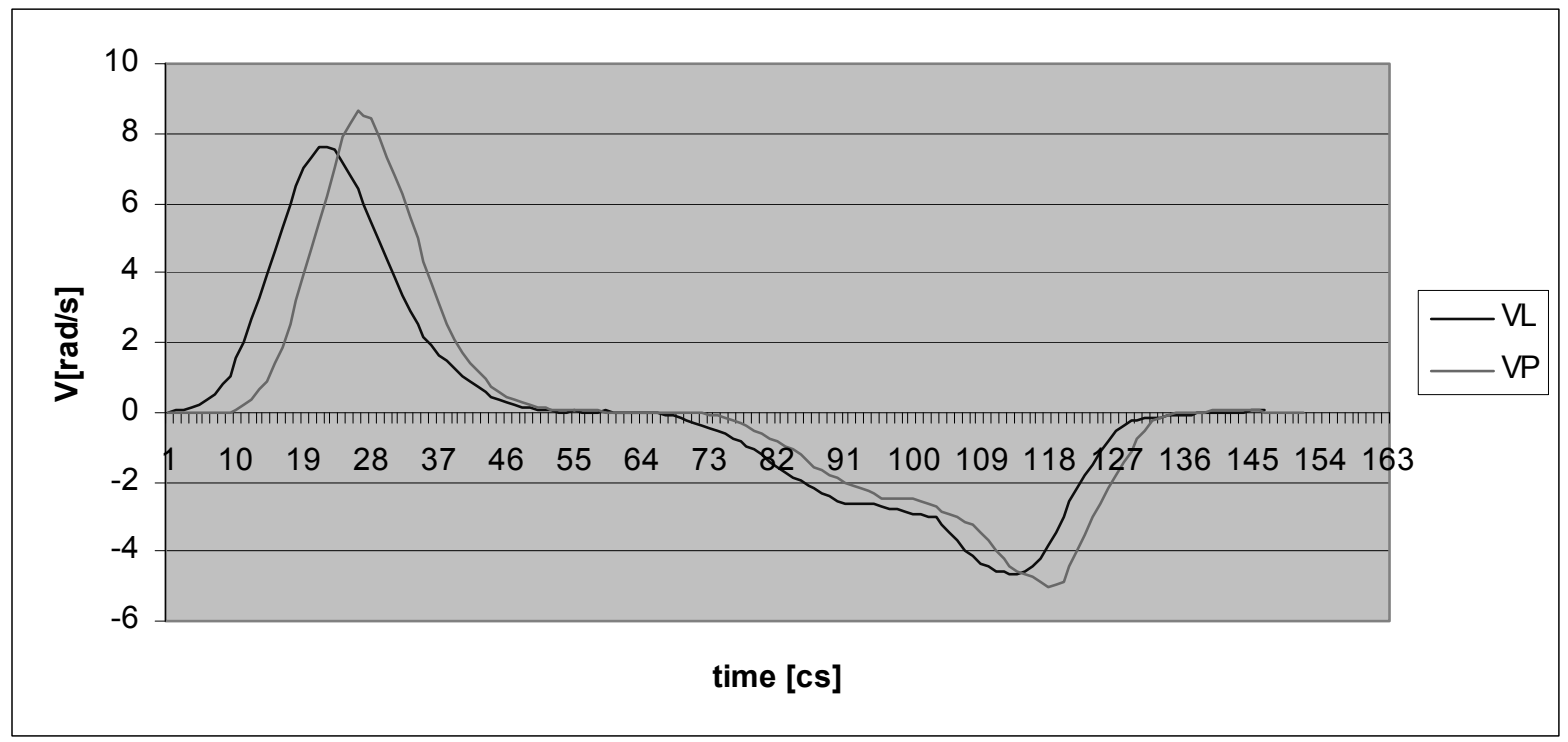

Fig. 1. A sample record of the change in angular velocity in the right (VP) and the left (VL) elbow joint with time variations during flexion and extension [rad/s]

Statistics. Empirical distributions of the analyzed characteristics did not differ significantly from the normal distribution, as determined with the Shapiro-Wilk test. An analysis of distributions at different barbell loads and time difference analysis was completed with t-test for dependent samples.

\section{Results}

Asymmetry of the downward and upward barbell motion was revealed by insignificant $(p<0.05)$ differences in the movement onset in the left and the right arm (Fig. 2). 


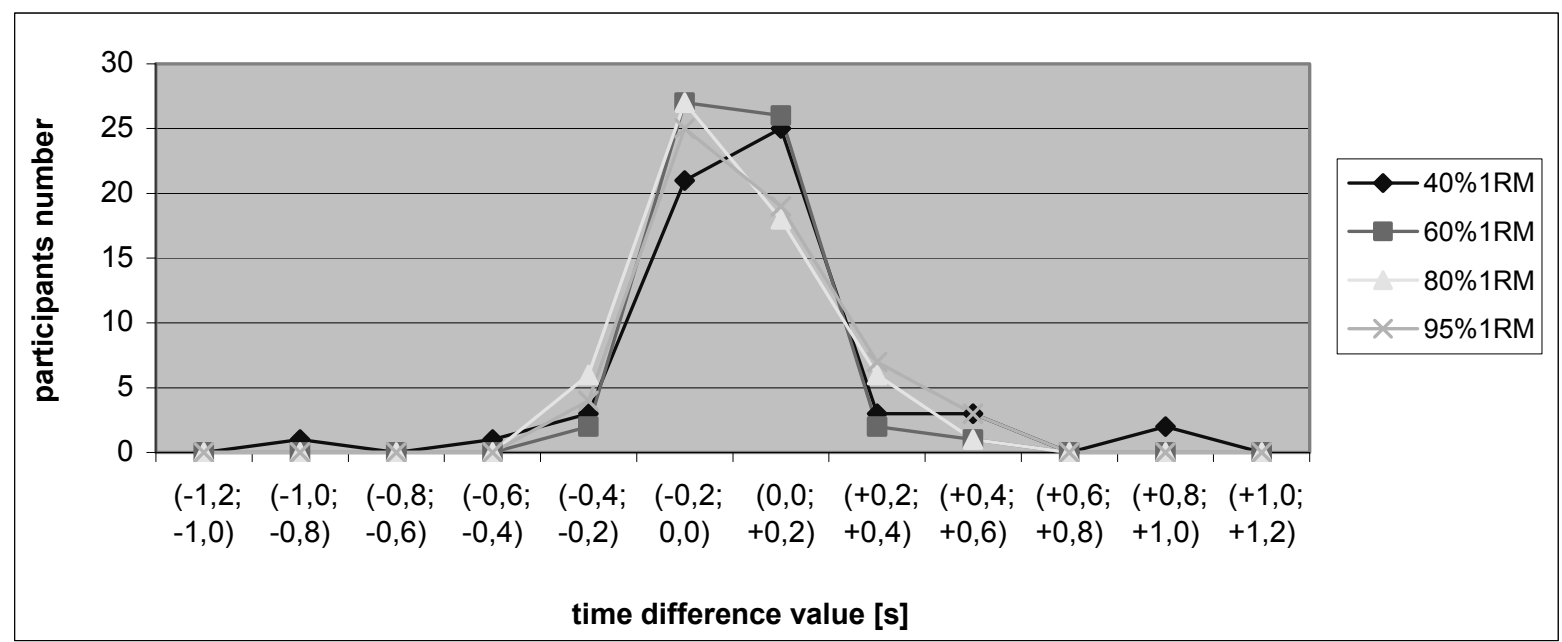

Fig. 2. Distribution of the time difference of flexion and extension movement onset in both upper limbs with $40 \% 1 \mathrm{RM}$ to $95 \% 1 \mathrm{RM}$

Time difference in achieving maximal angular velocity. During flexion most athletes achieved the maximal velocity at almost the same time point (Fig. 3), which was represented by insignificant time difference $(p<0.05)$.

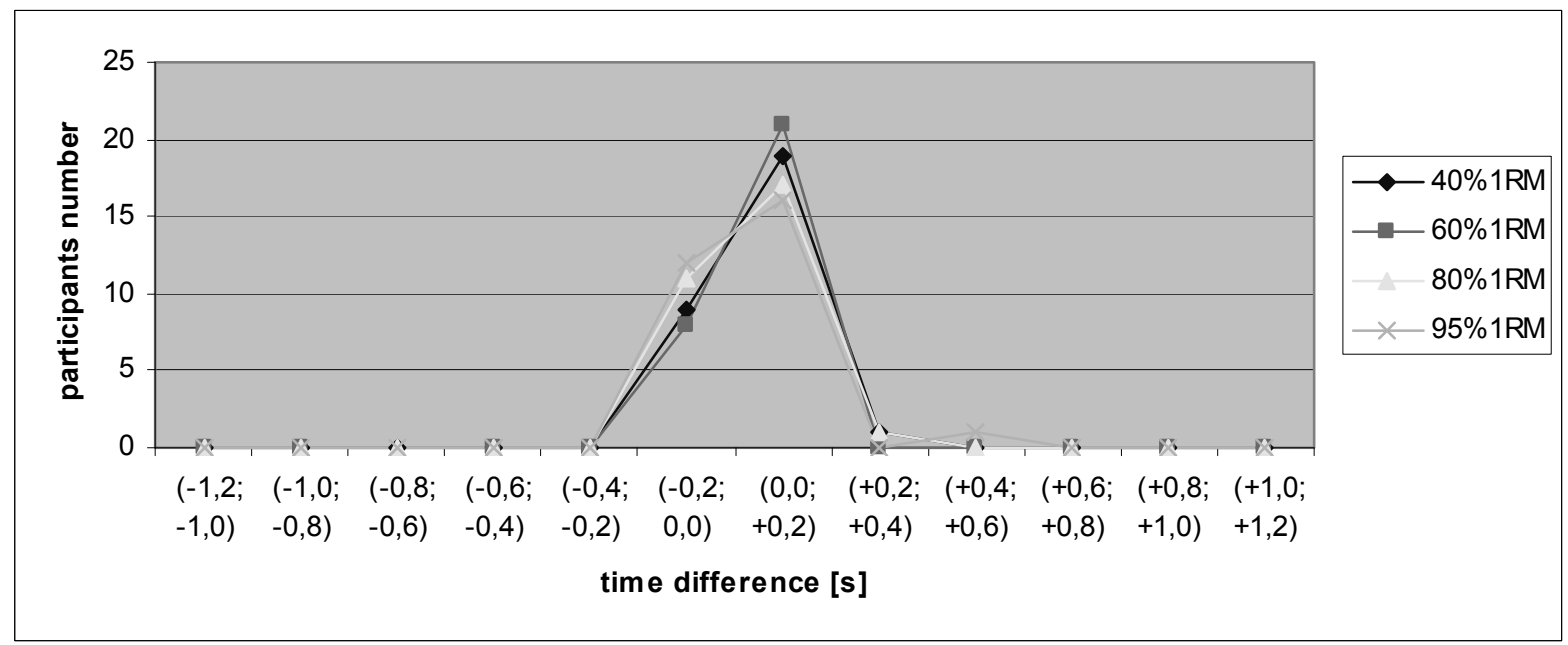

Fig. 3. Distribution of the time difference values for the maximum angular velocity during flexion in both upper extremities with $40 \%$ to $95 \% 1 \mathrm{RM}$

During extension the same general tendency was observed (Fig. 4). Also in this case, most of the subjects achieved the maximal velocity at approximately the same time point. However, with the increased barbell load (from $60 \%$ to $80 \% 1 \mathrm{RM}$ ) more athletes achieved the maximal velocity at different time points. These differences were statistically significant $(p<0.05)$. 


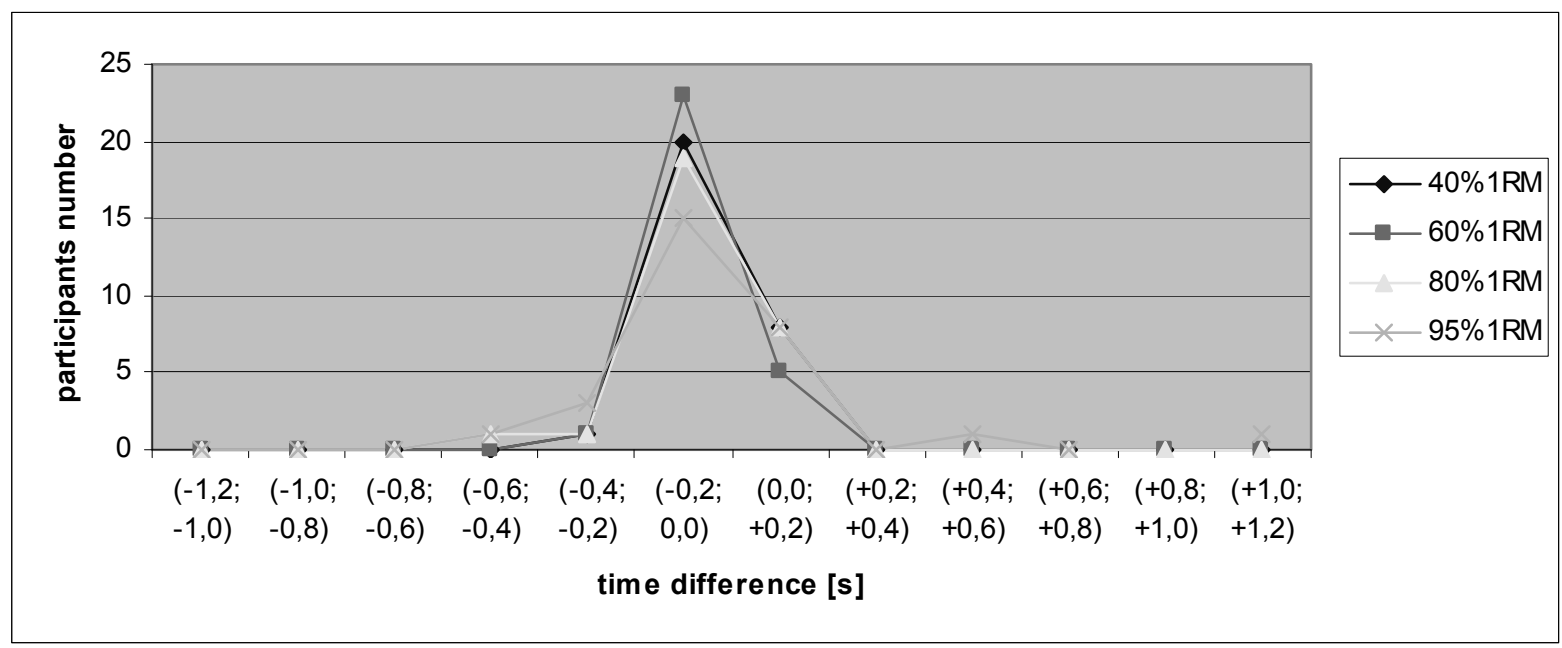

Fig. 4. Maximal angular velocity achievement time during extension in both upper limbs with $40 \%$ to $95 \%$ $1 \mathrm{RM}$

\section{Discussion}

The aim of the study was to determine the differences in flexion and extension movement onset and achievement of the maximal angular velocity in the elbow joint. These parameters did not change significantly with the increased barbell load. It suggests that most movements were performed symmetrically. However, based on the available studies, it could have been suspected that asymmetry would increase with the load [3].

In these studies, the primary criterion of symmetry assessment was the difference in the motion onset and achievement of maximal angular velocity in both elbows joints. It means that the beginning of movement and achievement of maximal angular velocity should appear simultaneously in the right and left upper limb.

With the increased load the asymmetry increased neither during flexion nor extension. In only a few cases differences of flexion and extension onset were significant (more than one second - with $40 \% 1 \mathrm{RM}$ ). This does not mean that with the least load the asymmetry was the greatest but only that two subjects started the movement this way.

The maximal angular velocity achievement time was similar in both upper limbs. A tendency (statistically insignificant) towards asymmetry was observed with (or more) 60\% 1RM. However, the symmetry of the extension movement deteriorated with the increasing load. Most athletes achieved the maximal angular velocity at almost the same time point; however, the differences between $60 \% 1 \mathrm{RM}$ and $80 \% 1 \mathrm{RM}$ were significant. A similar phenomenon (although statistically insignificant) was observed between $80 \% 1 \mathrm{RM}$ and 95\% 1RM. So during extension subjects performed the bench press in a symmetrical manner up to $60 \%$ of $1 \mathrm{RM}$. With greater loads the asymmetry increased, which could be explained by the fact that muscle tension decreased with the increasing load, and subsequently movements were performed with lower tension of synergistic muscles. This meant a lower number of excited motor units, resulting in less accurate movement control.

Based on this observation, a question arises why the eccentric movement, which is more difficult in control, was performed more symmetrically than the concentric one? In this case, it was probably related to a greater difficulty in accomplishing the upward motion. During this phase, athletes had to generate maximal strength. Whereas the downward movement with the gravity does not require so much muscle activity, and therefore the asymmetry increased significantly only during extension.

It can be concluded that if the goal of the training process is to improve technique or any other motor skill using greater loads, it would be more efficient to do that using downward movement. More generally, it could be stated that working on technique would be more effective in eccentric exercise. However, it should be noted that only two repetitions of an eccentric exercise with the 
maximal load can cause a significant decrease in muscle strength [1]. In concentric movements the greatest efficiency will be with the load up to $60 \% 1 \mathrm{RM}$.

\section{Conclusions}

1. The flexion movement was performed symmetrically independently of the barbell load.

2. The extension movement was performed symmetrically only with $40 \% 1 \mathrm{RM}$, with $60 \%$ $1 \mathrm{RM}, 80 \% 1 \mathrm{RM}$ and 95\%1RM the movement was asymmetrical.

\section{References}

1. Borniquez C, Crivellini M, Divieti L, Galli M. Mental stress influence on movement. In: Barabos A, Fabian G, eds. Biomechanics in Sport. Budapest: Hungarian University of Physical Education; 1995, 65-68.

2. Carpes FP, Bini RR, Mota CB. Training level, perception and bilateral asymmetry during multi-joint legpress exercise. Braz J Biomotricity. 2008;2:51-62.

3. Dias RMR, Cyrino ES, Salvador EP, et al. Influence of familiarization process on muscular strength assessment in 1-RM test. Revista Brasileira de Medicina do Esporte. 2005;11(1):39-42.

4. Dohoney P, Chromiak JA, Lemire D, Abadie BR, Kovacs C. Prediction of one repetition maximum (1RM) strength from a 4-6 RM and 7-10 RM submaximal strength test in healthy young adult males. J Exerc Physiol 2002;5(3):54-59.

5. Hakkinen K, Kraemer WJ, Newton RU. Muscle activation and force production during bilateral and unilateral concentric and isometric contractions of the knee extensors in men and women at different ages. Electromyogr Clin Neurophysiol. 1997;37:131-142.

6. Howard JD, Enoka RM. Maximum bilateral contractions are modified by neurally mediated interlimb effects. J Appl. Physiol. 1991;70:306-316.

7. Kawakami Y, Sale DG, MacDougall JD, Moroz JS. Bilateral deficit in plantar flexion: relation to knee joint position, muscle activation, and reflex excitability. Eur J Appl Physiol. 1998;77:212-216.

8. Koh TJ, Grabiner MD, Clough CA. Bilateral deficit is larger for step than for ramp isometric contractions. J Appl Physiol. 1993;74:1200-1205.

9. Migasiewicz J, Wiesner W, Seidel W, et al. Analysis of selected parameters of powerlifting technique of disabled competitors. Polish Journal of Environmental Studies. 2006;(15)2:603-606.

10. Nosaka K, Sakamoto K. The repeated bout effect of reduced-load eccentric exercise on elbow flexor muscle damage. Eur J Appl Physiol. 2001;85(1-2):34-40.

11. Oda S, Moritani T. Maximal isometric force and neural activity during bilateral and unilateral elbow flexion in humans. Eur J Appl Physiol. 1994;69:240-243.

12. Owings TM, Grabiner MD. Fatigue effects on the bilateral deficit are speed dependent. Med Sci Sports Exerc. 1998;30(8):1257-1262.

13. Seki T, Ohtsuki T. Influence of simultaneous bilateral exertion on muscle strength during voluntary submaximal isometric contraction. Ergonomics. 1990;33:1131-1142.

14. Smak W, Neptune RR, Hull ML. The influence of pedaling rate on bilateral asymmetry in cycling. J Biomech. 1999;32(9):899-906.

15. Vint PF, Hinrichs RN. Differences between one-foot and two-foot vertical jump performances. J Appl Biomech. 1996;12:338-358. 